Обоснование. Ожирение считается одной из самых распространенных неинфекционных болезней. Избыточная масса тела и ожирение негативно влияют на все сферы человеческой деятельности, приводя к развитию сопутствующих заболеваний и потере трудоспособности.

Цель. Оценить распространенность избыточной массы тела и ожирения среди взрослого населения Ярославской области в зависимости от пола и возраста.

Memoды. Работа выполнена по результатам комплексных медицинских обследований 13948 лиц обоего пола в возрасте от 20 до 79 лет, постоянно проживающих на территории Ярославской области. Антропометрическое обследование проводили по стандартным методикам с измерением массы тела и длины тела, с последующим расчетом индекса массы тела. Избыточной считали массу тела при индексе 25,0-29,9 кг/м², ожирение - $\geq 30$ кг/м². Содержание жировой ткани определяли методом биоимпедансометрии.

Результаты. Распространенность избыточной массы тела среди взрослого населения Ярославской области составила 34,2\%, ожирения - 31,6\%. Предожирение в 1,5 раза чаще встречалось среди мужчин, ожирение в 1,3 раза чаще регистрировали у женщин. Распространенность ожирения I степени составила 20,9\%, II и III степени - 7,9\% и 2,8\% соответственно. Доля людей с избыточной массой тела и ожирением увеличивалась с возрастом, достигая максимума к 50-59 годам для мужчин и 60-69 годам для женщин. Градиент возрастных изменений более выражен у женщин. У обследованных с нормальными значениями индекса массы тела встречаемость избыточного жироотложения составила $38 \%$.

Заключение. Проведенное исследование показало высокую степень распространенности избыточной массы тела и ожирения среди взрослого населения Ярославской области.

КЛЮЧЕВЫЕ СЛОВА: ожирение; избыточная масса тела; индекс массы тела; взрослое население; Ярославская область.

\title{
PREVALENCE OF OVERWEIGHT AND OBESITY AMONG THE ADULT POPULATION OF THE YAROSLAVL REGION
}

\author{
(c) Nataliya N. Tyatenkova*, luliya E. Uvarova
}

P.G. Demidov Yaroslavl State University, Yaroslavl, Russia

BACKGROUND: Obesity is one of the most common non-communicable diseases. Overweight and obesity negatively affect all spheres of human activity, leading to the development of related diseases and disability.

AIMS: to estimate the prevalence of overweight and obesity among the adult population of the Yaroslavl region according to sex and age.

MATERIALS AND METHODS: The research included results of comprehensive medical examinations of 13948 persons of both sexes aged from 20 to 79 permanently residing in the Yaroslavl region. Anthropometric examination was performed by standard methods with the measurement of body weight and body length and calculation of body mass index. Overweight was defined as having a BMI to $25.0-29.9 \mathrm{~kg} / \mathrm{m}^{2}$, obesity was defined as having BMI grater or equal to $30 \mathrm{~kg} / \mathrm{m}^{2}$. The fat compound of body mass was determined by bioimpedancemetry.

RESULTS: The prevalence of overweight among the adult population of the Yaroslavl region was 34.2\%, obesity was $31.6 \%$. Overweight in men of all ages was at least 1.5 times more prevalent than women, obesity in women was 1.3 times more prevalent than in men. The prevalence of first-degree obesity was $20.9 \%$, grade II and III $-7.9 \%$ and $2.8 \%$, respectively. The proportion of overweight and obesity increased with age, reaching a maximum of 50-59 years for men and 60-69 years for women. The gradient of age changes is more pronounced in women. Patients with normal body mass index had excessive fat deposition in $38 \%$ of cases.

CONCLUSIONS: The study showed a high prevalence of overweight and obesity among adults in the Yaroslavl region.

KEYWORDS: obesity; overweight; body mass index; body mass distribution; adult. 


\section{ОБОСНОВАНИЕ}

В XXI веке ожирение считается одной из главных неинфекционных болезней, захлестнувшей всё человечество. Только за период с 1980 по 2013 гг. число взрослых людей с ожирением увеличилось на 27,5\% [1]. По данным BO3, на 2016 г. в мире насчитывалось более 1,9 млрд людей старше 18 лет, имеющих избыточную массу тела, из них у 650 млн диагностировали ожирение [2]. В России избыточная масса тела отмечается у 59,8\% взрослого населения, ожирение - у 26,5\% [3].

Избыточная масса тела и ожирение негативно влияют на все сферы человеческой деятельности, приводя к развитию сопутствующих заболеваний и потере трудоспособности. С избыточной массой тела и ожирением, в том числе и скрытым, ассоциированы такие заболевания, как сахарный диабет [4], метаболический синдром, артериальная гипертензия, нарушение репродуктивной функции [5]. Ведущими причинами ожирения считают нерациональное питание и низкую физическую активность.

\section{ЦЕЛЬ}

Оценить распространенность избыточной массы тела и ожирения среди взрослого населения Ярославской области в зависимости от пола и возраста.

\section{МЕТОДЫ}

\section{Дизайн исследования}

Проведено обсервационное одноцентровое одномоментное выборочное неконтролируемое исследование.

\section{Критерии соответствия}

Критерии включения: выборка формировалась из числа лиц в возрасте от 20 до 79 лет включительно, постоянно проживающих на территории Ярославской области.

Другие критерии включения и невключения не применялись.

\section{Условия проведения}

В исследовании приняли участие пациенты Ярославского регионального Центра здоровья на базе ГБУз ЯО «ЯОКГВВ-МЦ «Здоровое долголетие».

Продолжительность исследования

Исследование проведено в период с января 2010 по февраль 2018 гг.

Таблица 1. Классификация категорий ИМТ по данным ВОЗ (2000)
Описание медицинского вмешательства

Проводилось антропометрическое обследование с измерением массы тела и длины тела по стандартным методикам и расчетом индекса массы тела (ИМТ). Для выявления случаев избыточного жироотложения при нормальных значениях индекса массы тела измеряли содержание жировой массы методом биоимпедансометрии.

\section{Основной исход исследования}

В качестве основного результата исследования использовали показатели распространенности избыточной массы тела и ожирения с учетом возраста и пола.

\section{Анализ в подгруппах}

Выборка была поделена по возрастным декадам с учетом половой принадлежности.

Методы регистрации исходов

Антропометрическое обследование проводили по стандартным методикам с измерением массы тела и длины тела. Индекс массы тела (ИМТ) рассчитывали по формуле: ИМТ=масса тела (кг) / длина тела $\left(\mathrm{M}^{2}\right)$. Массу тела оценивали в зависимости от величины ИМТ (табл. 1), используя классификацию ВОЗ [6].

Для выявления случаев избыточного жироотложения при нормальных значениях индекса массы тела измеряли и оценивали содержание жировой массы методом биоимпедансометрии с использованием анализатора состава тела АВС-01 «Медасс» (НТЦ «Медасс», Москва). Результаты заносились в электронную базу данных.

\section{Этическая экспертиза}

Исследование прошло этическую экспертизу и одобрено Локальным этическим комитетом на базе ФГБОУ ВО «Ярославский государственный университет им. П.Г. Демидова» (заключение от 15.12.2015 г.). Все пациенты, принявшие участие в обследовании, дали информированное согласие.

\section{Статистический анализ}

Принципы расчета размера выборки: Размер выборки предварительно не рассчитывался.

Методы статистического анализа данных: статистический анализ проводили с помощью программы Statistica 10.0 и Microsoft Excel 2010. Для изучаемых параметров рассчитывали среднее арифметическое (M), ошибку среднего (m), стандартное отклонение (б). Для оценки статистической значимости межгрупповых различий использовали U-критерий Манна-Уитни при $\mathrm{p}<0,05$. Для описания качественных признаков применяли относительную частоту (\%).

\begin{tabular}{|c|c|}
\hline Значения ИМТ, кг/м² & Классификация категорий \\
\hline $18,50-24,99$ & Нормальная масса тела \\
\hline $25,00-29,99$ & Предожирение (Избыточная масса тела) \\
\hline $30,00-34,99$ & Ожирение I степени \\
\hline $35,00-39,99$ & Ожирение II степени \\
\hline 40,00 и более & Ожирение II степени \\
\hline
\end{tabular}


Таблица 2. Характеристика обследованного контингента

\begin{tabular}{lcccccc}
\hline & \multirow{6}{*}{ Пол } & \multicolumn{5}{c}{ Количество обследованных, чел. } \\
\cline { 2 - 7 } & $\mathbf{2 0 - 2 9}$ лет & $\mathbf{3 0 - 3 9}$ лет & $\mathbf{4 0 - 4 9}$ лет & $\mathbf{5 0 - 5 9}$ лет & $\mathbf{6 0 - 6 9}$ лет & 70-79 лет \\
\hline $\mathrm{M}$ & 590 & 535 & 590 & 649 & 578 & 201 \\
Ж & 1540 & 1383 & 1590 & 2458 & 2600 & 1234 \\
Bce & 2130 & 1918 & 2180 & 3107 & 3178 & 1435 \\
\hline
\end{tabular}

\section{РЕЗУЛЬТАТЬ}

Объекты (участники) исследования

Работа выполнена по результатам комплексных медицинских обследования 13948 лиц обоего пола (табл. 2), из них 3143 мужчин (22,5\% от общего числа обследованных) и 10805 женщин $(77,5 \%)$.

\section{Основные результаты исследования}

В обследованной популяции среднее значение ИМТ без учета возраста и пола составило 27,6 кг/м², что в соответствии с критериями ВОЗ относится к избыточной массе тела (предожирению). Среди лиц, прошедших обследование, 2,6\% и 31,6\% имели недостаточную и нормальную массу тела соответственно. Распространенность избыточной массы среди взрослого населения Ярославской области составила $34,2 \%$, ожирения $31,6 \%$.
Оценка по возрастным декадам показала, что у лиц 20-29 лет среднегрупповые значения ИМТ соответствовали нормальной массе тела, в группе 60-летних - ожирению I степени, в остальных возрастных группах предожирению. Доля лиц с нормальной массой тела с возрастом уменьшилась в 4 раза: с 60,8\% (20-29 лет) до 15,6\% (60-69 лет) и незначительно увеличивалась до 17,6\% в старшей возрастной группе (70-79 лет).

Анализ полученных результатов в половозрастных группах показал, что с возрастом наблюдалось достоверное увеличение ИМТ в обеих половых группах. В среднем ИМТ увеличился к 69 годам на $36 \%$ у женщин и на $16 \%$ у мужчин. ИМТ статистически значимо отличался между половыми группами во всех возрастах $(p<0,01)$. У мужчин 20-49 лет данный показатель выше по сравнению с женщинами, но после 50 лет ИМТ выше у женщин (табл. 3).

Распределение обследуемых по величине ИМТ представлено на рисунках 1 и 2. Наименьшая частота

Таблица 3. Значения ИМТ в половозрастных группах

\begin{tabular}{lcccccc}
\hline Возраст, & \multicolumn{2}{c}{ Все } & \multicolumn{2}{c}{ Мужчины } & \multicolumn{2}{c}{ Женщины } \\
\cline { 2 - 7 } годы & $\mathbf{M} \pm \mathbf{m}$ & $\boldsymbol{\sigma}$ & $\mathbf{M} \pm \mathbf{m}$ & $\boldsymbol{\sigma}$ & $\mathbf{M} \pm \mathbf{m}$ & $\boldsymbol{\sigma}$ \\
\hline $20-29$ & $23,0 \pm 2,11$ & 4,5 & $24,6 \pm 2,07$ & 4,3 & $22,3 \pm 2,07^{*}$ & 4,4 \\
$30-39$ & $25,6 \pm 2,28$ & 5,2 & $27,1 \pm 2,06^{+}$ & 4,2 & $25,0 \pm 2,33^{*+}$ & 5,4 \\
$40-49$ & $27,1 \pm 2,26$ & 5,1 & $28,1 \pm 2,07^{+}$ & 4,3 & $26,8 \pm 2,31^{*+}$ & 5,4 \\
$50-59$ & $29,3 \pm 2,34$ & 5,5 & $28,6 \pm 2,13^{+}$ & 4,6 & $29,5 \pm 2,39^{*+}$ & 5,7 \\
$60-69$ & $30,0 \pm 2,30$ & 5,3 & $28,6 \pm 2,10$ & 4,4 & $30,4 \pm 2,32^{*+}$ & 5,4 \\
$70-79$ & $29,6 \pm 2,25$ & 5,1 & $27,4 \pm 2,01^{+}$ & 4,1 & $29,9 \pm 2,27^{*}$ & 5,1 \\
\hline
\end{tabular}

* - статистически значимые отличия между мужчинами и женщинами $(\mathrm{p}<0,01)$

+ - статистически значимые отличия по сравнению с предыдущей возрастной группой того же пола $(p<0,05)$

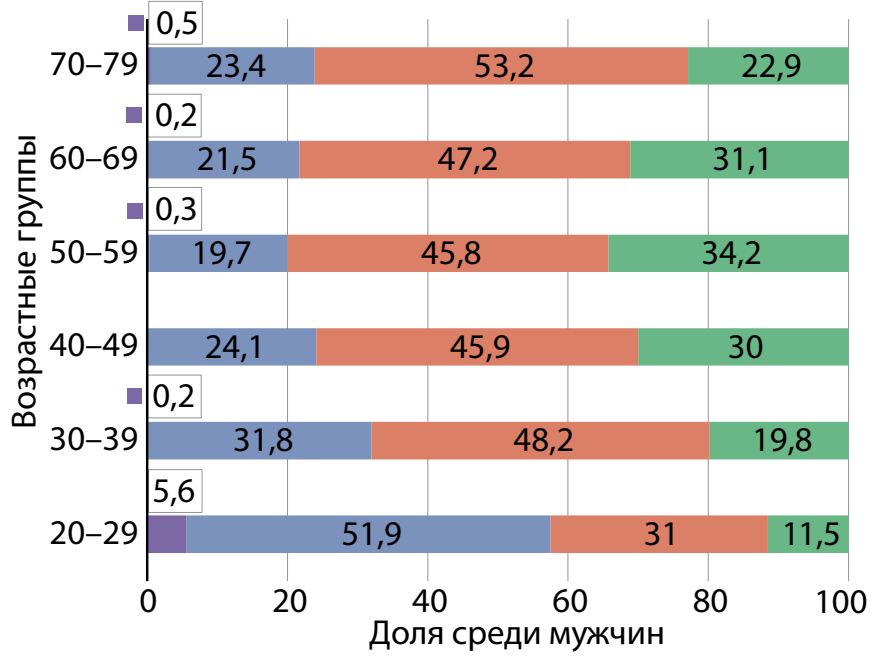

Дефицит массы тела Нормальная масса тела
Предожирение Ожирение

Рисунок 1. Распределение обследованных мужчин по величине индекса массы тела.

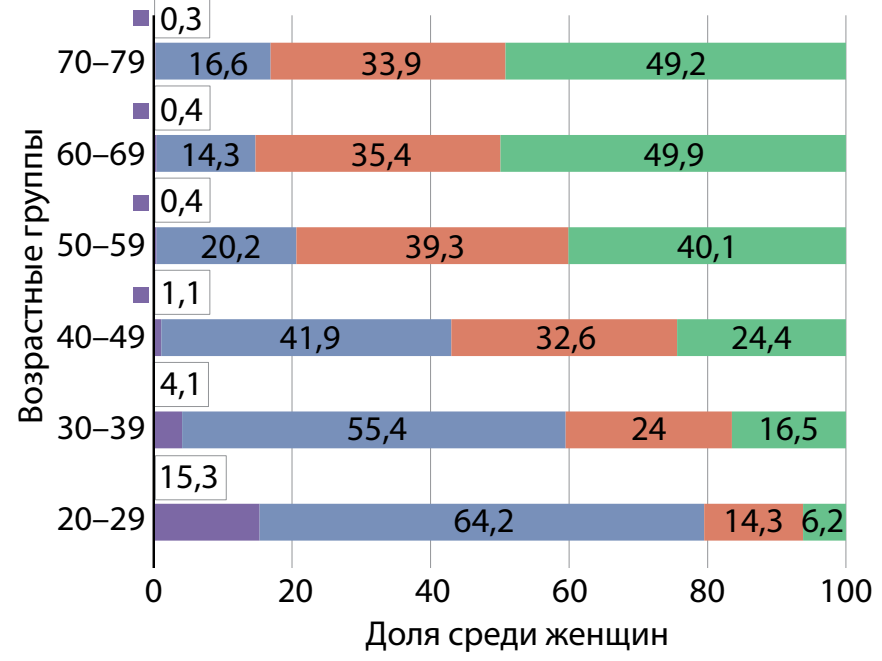

Дефицит массы тела Нормальная масса тела

Предожирение Ожирение

Рисунок 2. Распределение обследованных женщин по величине индекса массы тела. 
встречаемости избыточной массы тела и ожирения наблюдалась в самой молодой возрастной группе, максимальная - в группе 60-летних. С возрастом частота встречаемости лиц с нормальной массой тела снизилась в 2,2 раза у мужчин и в 3,8 раза среди женщин.

Избыточная масса тела в среднем в полтора раза чаще встречалась у мужчин (45,2\%) по сравнению с женщинами (29,9\%). С возрастом доля лиц с повышенной массой тела среди мужчин увеличилась в 1,7 раза, достигая своего максимума $(53,2 \%)$ в старшей возрастной группе (70-79 лет). У женщин частота встречаемости лиц с избыточной массой тела увеличилась в 2,3 раза, максимум наблюдался среди лиц 50-59 лет.

Частота встречаемости мужчин, страдающих ожирением, составила 24,9\%, женщин - 31,1\%. С возрастом их доля увеличилась в 6 раз, с 7,7\% до 46,7\%. Частота встречаемости мужчин с ожирением достигала своего максимума к 50 годам (34,2\%) и к 70 годам снизилась до 22,9\%. У женщин подобный максимум обнаружен у 60-летних и составил 49,9\%. При этом чаще всего встречались лица с ожирением I степени (20,9\%), на долю лиц с ожирением II и III степени приходилось 7,9\% и 2,8\% соот- ветственно. Ожирение I степени до 60 лет чаще встречалось среди мужчин, после 60 лет - у женщин (рис. 3, 4). Ожирение II и III степеней в 2 раза чаще встречалось среди женщин (7,8\% и 3\%), у мужчин - 4,9\% и 1,0\% соответственно.

Среди женщин с нормальным весом доля испытуемых с высокой жировой массой составила в среднем $33,2 \%$, с очень высокой - 9,9\%. Среди мужчин 10,7\% испытуемых с нормальным весом имели высокий уровень жира, 3,1\% - очень высокий. С возрастом частота встречаемости скрытого ожирения существенно менялась (рис. 5, 6).

Средний возраст в группе лиц с нормальной массой

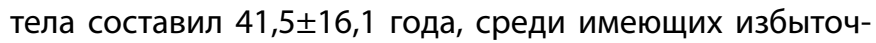

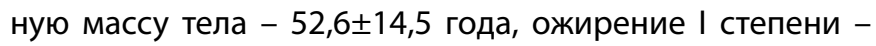
$56,8 \pm 13,3$ года, ожирение II степени - 57,6士11,9 года, ожирение III степени - 56,6 $\pm 11,8$ года (табл. 4). Большинство людей с ИМТ выше $25 \mathrm{kг} / \mathrm{M}^{2}$ представлены возрастной группой от 50 лет. Мужчины и женщины с нормальной массой тела не отличались по возрасту, в группах с избыточной массой тела и ожирением женщины были достоверно старше мужчин.
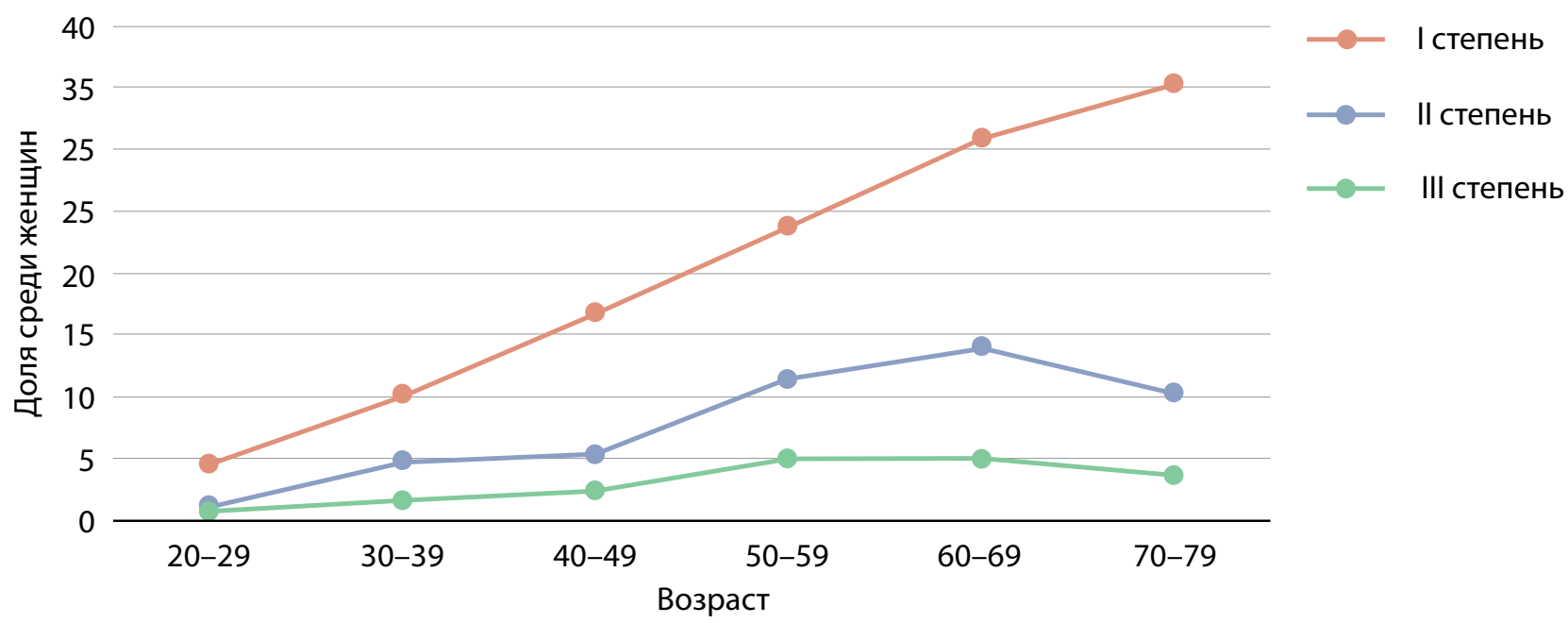

Рисунок 3. Частота встречаемости ожирения разных степеней у женщин 20-79 лет.

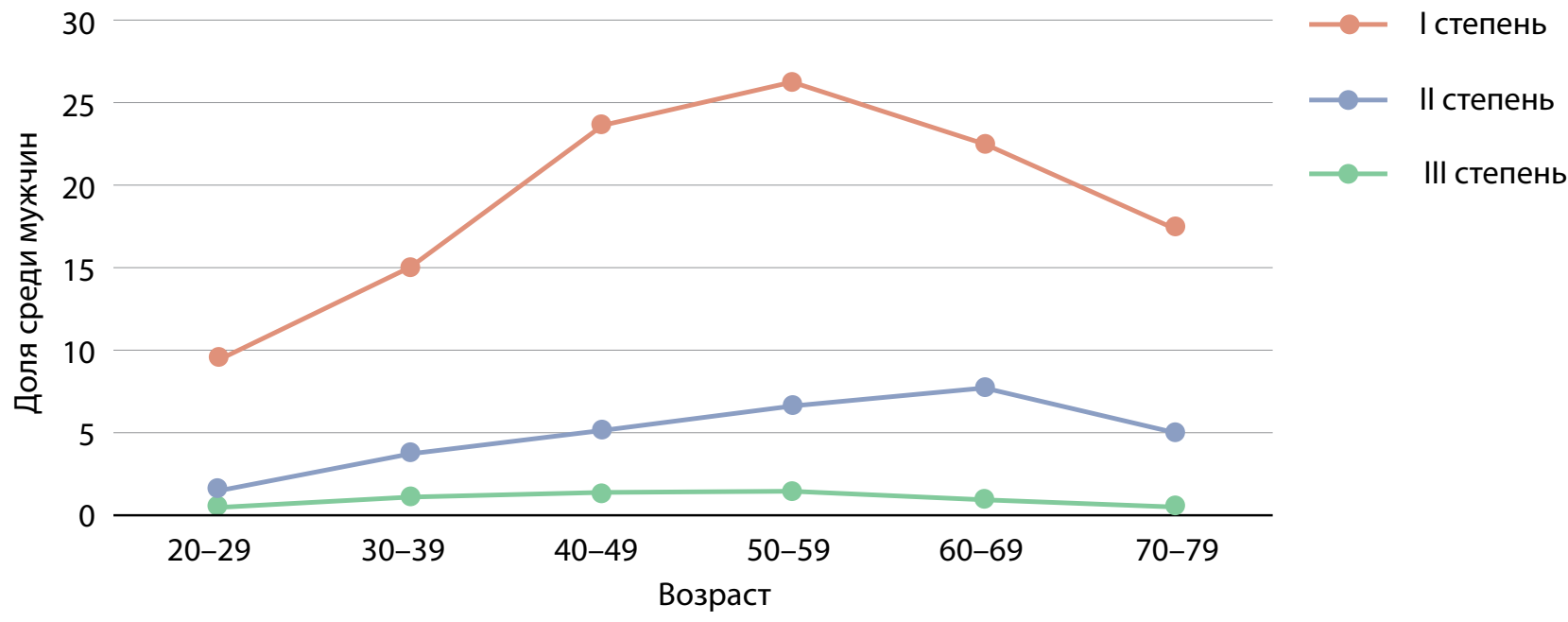

Рисунок 4. Частота встречаемости ожирения разных степеней у мужчин 20-79 лет. 


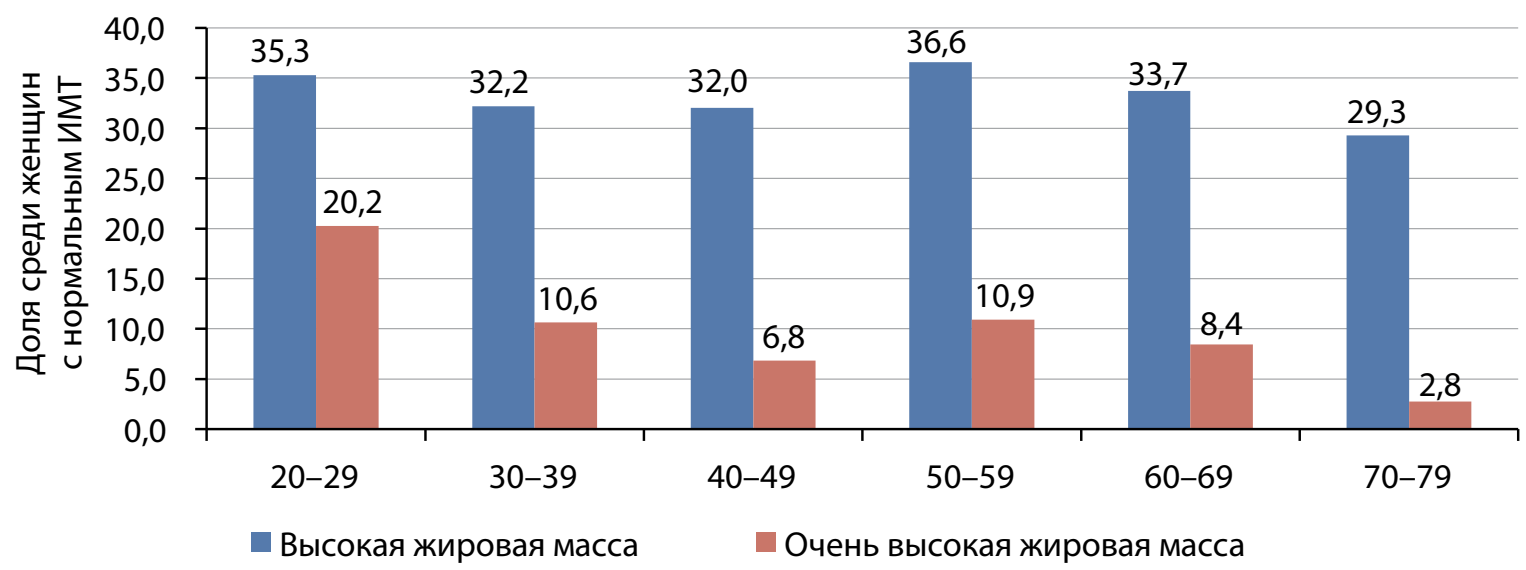

Рисунок 5. Распространенность повышенных значений жирового компонента у женщин с нормальным индексом массы тела.

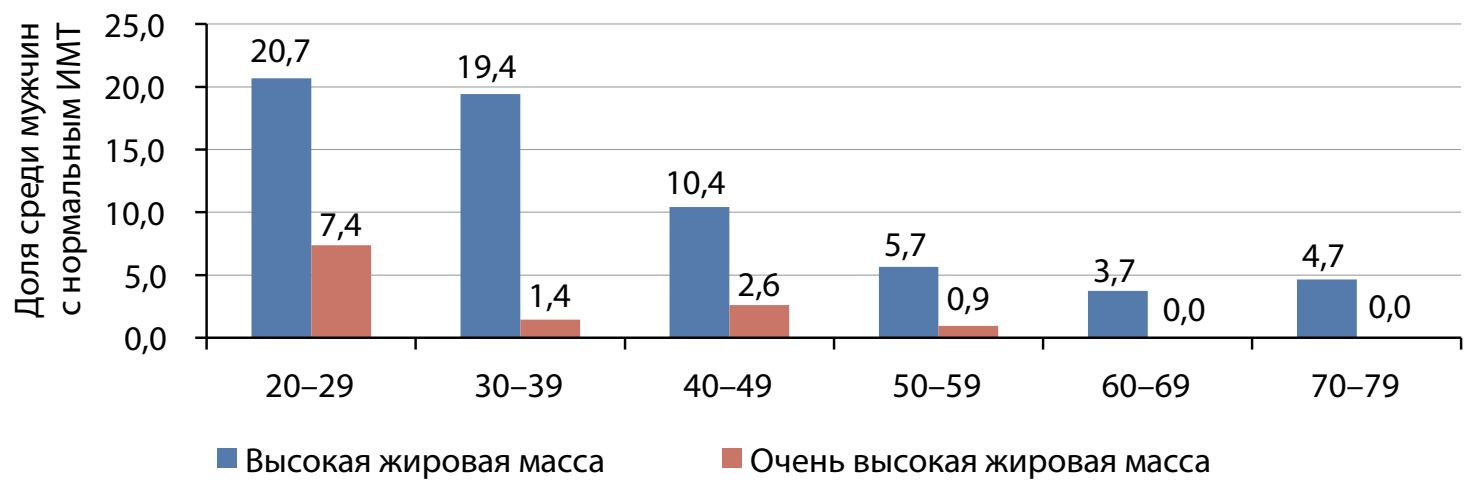

Рисунок 6. Распространенность повышенных значений жирового компонента у мужчин с нормальным индексом массы тела.

Таблица 4. Средний возраст в исследуемых группах в зависимости от ИМТ и пола

\begin{tabular}{cccc}
\hline Значения ИМТ & Все & Мужчины & Женщины \\
\hline $18,50-24,99$ & $41,5 \pm 16,1$ & $41,1 \pm 16,4$ & $41,6 \pm 16,0$ \\
$25,00-29,99$ & $52,6 \pm 14,5$ & $48,5 \pm 15,0$ & $54,3 \pm 13,9^{*}$ \\
$30,00-34,99$ & $56,8 \pm 13,3$ & $49,9 \pm 13,6$ & $58,6 \pm 12,6^{*}$ \\
$35,00-39,99$ & $57,6 \pm 11,9$ & $52,4 \pm 13,0$ & $58,5 \pm 11,4^{*}$ \\
40,00 и выше & $56,6 \pm 11,8$ & $48,6 \pm 12,1$ & $57,5 \pm 11,3^{*}$ \\
\hline
\end{tabular}

* - статистически значимые отличия в половых группах.

\section{ОБСУЖДЕНИЕ}

Результаты проведенного исследования показали высокую распространенность среди взрослого населения Ярославской области избыточной массы тела и ожирения. Результаты сопоставимы с данными, полученными при обследовании жителей г. Томска [7] и ниже по сравнению с таковыми по г. Иркутску [8]. По данным ВОЗ [3], в России доля лиц старше 20 лет с избыточной массой тела составляет 56,2\% среди мужчин и 62,8\% среди женщин, с ожирением - 18,6\% и 32,9\%. Исследования 2010-2012 гг. [9] показали, что таковых 61,5\% и 27,2\% соответственно. Разница в полученных результатах может быть связана с особенностями выборки и методами получения ростовесовых данных: различия в количестве и возрастном диапазоне испытуемых, получение данных путем опроса или антропометрическими измерениями и т.д. Не исключено также влияние социально-экономических факторов, различия в которых имеют место быть в разных регионах России и связаны с особенностями питания, проведения досуга, экономическим благополучием.

С возрастом происходит увеличение ИМТ на $36 \%$ у женщин и 16\% у мужчин. Общеизвестно, что уровень основного обмена и двигательная активность с возрастом снижаются при сохранившихся традициях питания. Исследования показывают [10], что взрослое население РФ отдает предпочтение малоподвижным формам досуга. Сочетание этих факторов неизбежно приводит к набору веса с возрастом. 
Избыточная масса тела у мужчин, проживающих в Ярославской области, встречалась в 1,5 раза чаще, но у женщин в 1,3 раза чаще диагностировали ожирение. Аналогичную особенность в распространении избыточной массы тела и ожирения регистрировали в ряде работ [7-9, 11, 12]. Градиент возрастных изменений ИМТ более выражен среди женского населения, что отмечалось другими исследователями [12]. Максимальный скачок в частоте встречаемости избыточной массы тела и ожирения у мужчин выявлен с 30-летнего возраста, что объясняется гиподинамией в связи с особенностями работы [13], предпочитаемым досугом [10] и семейной жизнью [14]. Аналогичный скачок в группе женщин происходит десятилетием позже и выражен сильнее, что может быть обусловлено не только вышеназванными причинами, но и наступлением менопаузы [15].

Важно отметить, что ИМТ не всегда отражает степень ожирения, особенно у пожилых людей с пониженной мышечной массой [16], поскольку не учитывает компонентный состав тела [17]. Среди населения Ярославской области с нормальным индексом массы тела методом биоимпедансометрии выявлено 30,5\% случаев высокой жировой массы и 7,2\% - очень высокой жировой массы. Таким образом, к диагностированной высокой доле людей с избыточной массой тела и ожирением стоит прибавить процент населения с так называемым скрытым ожирением. Проблема ожирения при нормальном весе обнаруживается во всех половозрастных группах и возникает при снижении доли скелетно-мышечной массы, ассоциированной с гиподинамией и особенностями питания.

Проблема избыточного веса и ожирения приняла статус эпидемии и оказывает влияние на социальноэкономические аспекты. В странах Европейского региона до 6\% расходов на здравоохранение приходятся на долю этой проблемы [18]. Затраты России на лечение и ведение пациентов, связанные с ожирением (острый инфаркт миокарда, острое нарушение мозгового кровообращения, сахарный диабет 2 типа), ежегодно составляют около 364,1 млрд рублей [19], а смертность взрослого населения вследствие избыточной массы тела и ожирения ежегодно составляет 3,4 млн человек [1].

К сожалению, ввиду ряда факторов, в долгосрочной перспективе ожидается рост уровня ожирения во всем мире [13]. Если тенденции останутся прежними, то к 2030 г. в Российской Федерации только от ожирения будут страдать 33\% мужчин и 26\% женщин [3].

\section{Ограничения исследования}

Стоит отметить, что данная работа была выполнена на основе выборки людей, пришедших на обследование в Ярославский региональный Центр здоровья, следовательно, срез населения, менее интересующийся состоянием своего здоровья и не посещающий медицинские учреждения с целью диагностики и профилактики, не затронут в исследовании. На репрезентативность выборки также могло сказаться неравномерное распределение обследованных в половых группах.

\section{ЗАКЛЮЧЕНИЕ}

Распространенность избыточной массы тела среди взрослого населения Ярославской области составила $34,2 \%$, ожирение - 31,6\%. Предожирение в 1,5 раза чаще встречалось среди мужчин, ожирение в 1,3 раза чаще регистрировали у женщин. Распространенность ожирения I степени составила 20,9\%, II и III степени - 7,9\% и 2,8\% соответственно. Доля людей с избыточной массой тела и ожирением увеличивалась с возрастом, достигая максимума к 50-59 годам для мужчин и 60-69 годам для женщин. Градиент возрастных изменений более выражен у женщин. У обследованных с нормальными значениями ИМТ встречаемость избыточного жироотложения составила $38 \%$.

\section{ДОПОЛНИТЕЛЬНАЯ ИНФОРМАЦИЯ.}

Источник финансирования. Подготовка и публикация рукописи проведены в рамках НИР ЯрГУ № ОП-2Г-13-2019.

Конфликт интересов. Авторы заявляют об отсутствии явных и потенциальных конфликтов интересов, связанных с публикацией настоящей статьи.

Участие авторов. Тятенкова Н.Н. - концепция и дизайн исследования, анализ и интерпретация данных, написание и редактирование текста; Уварова Ю.Е. - сбор и обработка материала, статистическая обработка данных. Оба автора внесли значимый вклад в проведение исследования и подготовку статьи, прочли и одобрили финальную версию статьи перед публикацией.

Благодарности. Авторы выражают благодарность сотрудникам Ярославского регионального центра здоровья за помощь в сборе фактического материала.

\section{СПИСОК ЛИТЕРАТУРЫ | REFERENCES}

1. Ng M, Fleming T, Robinson M, et al. Global, regional, and national prevalence of overweight and obesity in children and adults during 1980-2013: a systematic analysis for the Global Burden of Disease Study 2013. Lancet. 2014;384(9945):766-781. doi: https://doi.org/10.1016/S0140-6736(14)60460-8

2. World Health Organization [Internet]. Obesity and overweight. [cited 2019 May 8]. Available from: http://www.who.int/mediacentre/factsheets/fs311/en/.

3. Euro.who.int [Internet]. Russian Federation. [cited 2019 May 8]. Available from: http://www.euro.who.int/en/health-topics/diseaseprevention/nutrition/country-work/russian-federation2/

4. Бутрова С.А., Берковская М.А., Дзгоева Ф.Х., Комшилова К.А. Распространенность ожирения, кардиометаболических факторов риска, метаболического синдрома и сахарного диабета среди женщин различных возрастных групп Московского региона. // Ожирение и метаболизм. - 2009. - Т. 6. - №4. - С. 28-33. [Butrova SA, Berkovskaya MA, Dzgoeva FK, Komshilova KA. Rasprostranennost' ozhireniya,kardiometabolicheskikh faktorov riska,metabolicheskogo sindroma i sakharnogo diabetasredi zhenshchin razlichnykh vozrastnykh gruppMoskovskogo regiona. Obesity and metabolism. 2009;6(4):28-33. (In Russ.)] doi: https://doi.org/10.14341/2071-8713-4875

5. Горбатенко Н.В., Беженарь В.Ф., Фишман М.Б. Влияние ожирения на развитие нарушения репродуктивной функции уженщин (аналитический обзор литературы). // Ожирение и метаболизм. - 2017. - Т. 14. - №1 - С. 3-8. [Gorbatenko NV, Bezhenar VF, Fishman MB. Obesity and reproductive health of women. Obesity and metabolism. 2017;14(1):3-8. (In Russ.)] doi: https://doi.org/10.14341/omet201713-8 
6. World Health Organization. Obesity: preventing and managing the global epidemic. Report of a WHO Consultation. Geneva: WHO; 2000.

7. Кобякова О.С., Деев И.А., Бойков В.А., и др. Распространенность избыточной массы тела среди взрослого населения города Томска (по результатам анализа деятельности центров здоровья). // Медицина в Кузбассе. - 2014. - Т. 13. — № 2. C. 51-55. [Kobyakova OS, Deyev IA, Boykov VA et al. The prevalence of obesity among the adult population in Tomsk (results of analysis of the health centers). Medicine in Kuzbass. 2014;13(2):51-55. (In Russ.)]

8. Меньшикова Л.В., Бабанская Е.Б. Половозрастная эпидемиология ожирения. // Ожирение и метаболизм. — 2018. — Т. 15. — №2. C. 17-22. [Menshikova LV, Babanskaya EB. Age and sex epidemiology of obesity. Obesity and metabolism. 2018;15(2):17-22. (In Russ.)] doi: https://doi.org/10.14341/omet8782.

9. Руднев С.Г., Соболева Н.П., Стерликов С.А., и др. Биоимпедансное исследование состава тела населения России. - М.: РИО ЦНИИОИЗ; 2014. [Rudnev SG, Soboleva NP, Sterlikov SA, et al. Bioimpedance study of body composition in the Russian population. Moscow: PIO TSNIIOIZ; 2014. (In Russ.)]

10. Седова Н.Н. Досуговая активность граждан. // Социологические исследования. — 2009. — № 12. - C. 56-68. [Sedova NN. Leisuretime activities of Russian citizens. Sotsiol Issled. 2009;(12):56-68. (In Russ.)]

11. Бухарова Г.П., Романцова Т.И. Распространенность избыточного веса и ожирения по данным выборки московского региона. // Ожирение и метаболизм. - 2007. - Т. 4. — №2. C. 14-16. [Bukharova GP, Romantsova TI. Rasprostranennost' izbytochnogo vesa i ozhireniyapo dannym vyborki moskovskogo regiona. Obesity and metabolism. 2007;4(2):14-16. (In Russ.)] doi: https://doi.org/10.14341/2071-8713-5157

12. Шальнова С.А., Деев А.Д. Масса тела у мужчин и женщин (результаты обследования российской, национальной, представительной выборки населения). // Кардиоваскулярная терапия и профилактика. — 2018. - Т. 7. — №6. - С. 60-64.
[Shalnova SA, Deev AD. Body mass in men and women: the Russian national representative sample data. Cardiovascular therapy and prevention. 2008;7(6):60-64. (In Russ.)]

13. Philipson T, Posner RA. The long-run growth in obesity as a function of technological change. Perspect Biol Med. 2003;46(3 Suppl):S87-107. doi: https://doi.org/10.3386/w7423

14. Mata J., Frank R, Hertwiga R. Higher body mass index, less exercise, but healthier eating in married adults: Nine representative surveys across Europe. Soc Sci Med. 2015;138:119-127. doi: https://doi.org/10.1016/j.socscimed.2015.06.001

15. Bjortorp P. Abdominal obesity and the metabolic syndrome. Ann Med. 1992;24(6):465-468 doi: https://doi.org/10.3109/07853899209166997

16. Stenholm S, Harris TB, Rantanen T et al. Sarcopenic obesity: definition, cause and consequences. Curr Opin Clin Nutr Metab Care. 2008;11(6):693-700. doi: https://doi.org/10.1097/MCO.0b013e328312c37d

17. Blundell JE, Dulloo AG, Salvador J, Fruhbeck G. Beyond BMI - phenotyping the obesities. Obes Facts. 2014;7:322-328. doi: https://doi.org/10.1159/000368783

18. Всемирная организация здравоохранения. Европейская хартия по борьбе с ожирением. / Европейская министерская конференция ВОЗ по борьбе с ожирением. Питание и физическая активность в интересах здоровья; Ноябрь 15-17, 2006; Стамбул. [World Health Organization. European Charter on counteracting obesity. In: Proceedings of the WHO European Ministerial Conference on Counteracting Obesity. Diet and physical activity for health; 2006 Nov 15-17; Istanbul. (In Russ.)]

19. Крысанова В.С., Журавлева М.В., Дралова О.В., и др. Проблема ожирения и избыточной массы тела в Российской Федерации и ее фармакоэкономическая оценка. // Альманах клинической медицины. - 2015. - № S1. - C. 36-41. [Krysanova VS, Zhuravleva MV, Dralova OV, et al. The problem of obesity and overweight in the Russian Federation and its pharmacoeconomic assessment. Almanac of clinical medicine. 2015;(S1):36-41. (In Russ.)]

\section{ИНФОРМАЦИЯ ОБ АВТОРАХ [AUTHORS INFO]:}

*Тятенкова Наталия Николаевна, д.б.н., профессор [Nataliya N. Tyatenkova, PhD in biology, professor]; адрес: Россия, 150057, Ярославль, проезд Матросова, д. 9 [address: 9 Proyezd Matrosova 150057, Yaroslavl, Russia]; ORCID: http://orcid.org/0000-0001-8934-9986; eLibrary SPIN: 7746-6321; e-mail: tyat@bk.ru

Уварова Юлия Евгеньевна [luliya E. Uvarova]; ORCID: http://orcid.org/0000-0002-2983-6616; eLibrary SPIN: 9398-5898; e-mail: jyli_91@mail.ru

*Автор, ответственный за переписку / Corresponding author.

\section{ЦИТИРОВАТЬ:}

Тятенкова Н.Н., Уварова Ю.Е. Распространенность избыточной массы тела и ожирения среди взрослого населения Ярославской области // Ожирение и метаболизм. - 2020. - Т. 17. - №2. - С. 164-170. doi: https://doi.org/10.14341/omet10284

\section{TO CITE THIS ARTICLE:}

Tyatenkova NN, Uvarova IE. Prevalence of overweight and obesity among the adult population of the Yaroslavl region. Obesity and metabolism. 2020;17(2):164-170. doi: https://doi.org/10.14341/omet10284 